\title{
A Novel Validated Stability-indicating HPTLC Method to Quantitate Forskolin as a Bulk Drug and in a Nanosuspension
}

\author{
NAZIA KHAN*, AMEEDUZZAFAR ${ }^{1}$, A. ALI AND F. J. AHMAD \\ Department of Pharmaceutics, Faculty of Pharmacy, Jamia Hamdard, New Delhi-110 062, India, ${ }^{1}$ College of Pharmacy, Aljouf \\ University, Sakaka, Aljouf, KSA
}

\author{
Khan et al.: Quantification of Forskolin by a Novel Validated HPTLC Method
}

\begin{abstract}
A novel, reproducible, precise, accurate and dependable high-performance thin-layer chromatographic technique was developed and authenticated as per International Conference on Harmonization guiding principle for quantitative evaluation of forskolin as a bulk drug and in a nanosuspension. Chromatographic plates 60 F-254 pre-coated with silica gel and a solvent system of ethyl acetate:hexane:formic acid (7:2.9:0.1 v/v) was used for the development of the plates. Densitometric scanning of the developed plates in the reflectance mode at $555 \mathrm{~nm}$ using a Camag TLC scanner after exposing the plates to anisaldehyde-sulphuric acid reagent, gave a compact spot for forskolin $\left(R_{\mathrm{f}}=\mathbf{0 . 4 6} \pm \mathbf{0 . 0 1 2}\right)$. In accordance to the $\mathrm{ICH}$ guidelines, the method was validated for linearity, precision (intra- and inter-day), limit of quantification and limit of detection, robustness and accuracy. Good linear relationship was observed for the calibration plots with linear range from $100-1000 \mathrm{ng} / \mathrm{spot}$ and a coefficient of correlation $\mathbf{r}^{2}=0.994$. Limit of detection and limit of quantification were found to be $\mathbf{1 0 . 8 3}$ and $36.12 \mathrm{ng} / \mathrm{spot}$, respectively. The method appeared to be well suited for determining forskolin content in the developed nanoformulation. Forskolin was found to be susceptible to acid and alkali hydrolysis, oxidation, photochemical degradation with UV and sunlight. Statistical analysis confirmed that the process developed is precise, repeatable, accurate, and suitable for the analysis of forskolin in a formulation and could be used also for analysis of forskolin in plasma and other biological fluids. The ability of the developed method to separate the drug from its degradation products effectively confirmed that it could be a stability indicating method.
\end{abstract}

Key words: Forskolin, HPTLC, ICH, nanosuspension, validation

Herbal medicines are the oldest form of healthcare. According to World Health Organization (WHO), plants are the major sources for the drugs to be prescribed globally and constitute around $25 \%$ and currently 121 compounds with therapeutic potential are actively used. WHO listed a total 252 drugs, which are considered essential and basic, of which $11 \%$ are actually of plant origin ${ }^{[1]}$. Chemically, forskolin is $7 \beta$-acetoxy8,13 -epoxy- $1 \alpha, 6 \beta, 9 \alpha$-trihydroxy-labd-14-en-11-one ${ }^{[2]}$ (fig. 1). It is a diterpenoid isolated from Coleus forskolii belonging to the family Lamiaceae and been previously reported to have antiglaucoma potential ${ }^{[3]}$. Forskolin is a lipophilic compound that when applied topically to the eye for treating glaucoma it acts by stimulating adenylate cyclase of ciliary epithelium to increase levels of cyclic adenosine monophosphate leading reduced aqueous humor inflow and a reduction in the intraocular pressure $(\mathrm{IOP})^{[4-6]}$. Increased IOP resulting

*Address for correspondence E-mail: khannaz88@rediffmail.com in optic nerve damage is primarily responsible for impairment in visualisation leading to glaucoma. It is basically an irreversible cause of blindness, however if detected, can be treated well with medications ${ }^{[7]}$. Forskolin is one of the herbal drug, which was found to have antiglaucoma potential and been used for treating glaucoma ${ }^{[3]}$. Of all the chromatographic techniques, high-performance thin-layer chromatography (HPTLC) is one of the significant planar chromatographic technique, which is widely used for the quantification of various traditional and other forms of therapeutic

This is an open access article distributed under the terms of the Creative Commons Attribution-NonCommercial-ShareAlike 3.0 License, which allows others to remix, tweak, and build upon the work non-commercially, as long as the author is credited and the new creations are licensed under the identical terms

Accepted 21 July 2018

Revised 26 December 2017

Received 01 June 2017

Indian J Pharm Sci 2018;80(5):820-826 
www.ijpsonline.com

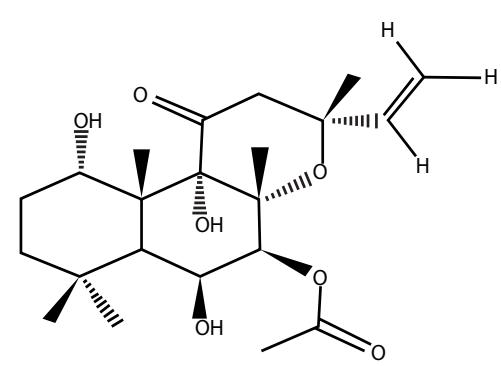

Fig. 1: Structure of forskolin

agents. Rapid cost-effective analysis of simple mixtures, ability to analyse various samples on a single plate run with efficient quantification ${ }^{[8]}$ are some of the outstanding features of HPTLC that make it different from other chromatographic techniques. Requirement for very low quantity of mobile phase along with ease in preparation of samples for calibration plot are other advantages of HPTLC analysis of drugs. HPTLC as well as other chromatographic techniques such as HPLC, gas chromatography mass spectrometry (GC/MS) have previously being reported for forskolin determination in different formulations and natural fluids ${ }^{[9,10]}$. However, the present method developed and validated is different from the other described methods as it has been used for the analysis of drug content in the developed nanosuspension, which has not been reported till now.

The efficacy and safety of a pharmaceutical product depended a lot on the chemical behaviour of the drug molecule. Forced or stress degradation studies are basically conducted at different stress or accelearted conditions, probably thought to be more severe for a particular drug molecule to assess its degradation pattern and hence, shelf-life. Stress testing is intended for degradation product identification with simultaneous determination of inherent stability of the active compounds as mentioned in the International Conference on Harmonization (ICH) guidelines. Various tests and parameters need to be tested for stress degradation studies with minimal factors like susceptibility of the compound (solid or in liquid and suspension form) to oxidation, acid and base hydrolyis, thermal and photolytic degradation have been suggested ${ }^{[11-14]}$.

In the present investigation, a novel HPTLC method was developed and validated as per the guidelines of $\mathrm{ICH}$ for determination of forskolin as a bulk drug and in a pharmaceutical dosage form. Stress degardation studies were performed to assess the stability of forskolin.

\section{MATERIALS AND METHODS}

Sami Labs, Bangalore, India, provided a gift sample of forskolin. The plates for TLC analysis used were pre-coated silica gel aluminium plates (E. Merck, Darmstadt, Germany) procured from Anchrom Technologies, Mumbai, India. All the reagents and chemicals used were of analytical grade and were procured from S.D. Fine-Chem Ltd., Mumbai, India.

\section{TLC instrumentation and conditions:}

The samples for chromatographic analysis comprising of the standards and forskolin extracted samples were spotted on a $60 \mathrm{~F} 254(10 \times 10 \mathrm{~cm}$ with $250 \mu \mathrm{m}$ thickness) aluminium plate precoated with silica gel with a sample applicator Camag Linomat IV (Camag, Muttenz, Switzerland) and a Hamilton syringe of $100 \mu \mathrm{l}$ capacity. Prior to application of spots on TLC plates, plate's pre-treatment and activation was done at $60 \pm 5^{\circ}$ in a hot air oven to make them absolute moisture free for about 5 to $10 \mathrm{~min}$ so as to obtain better compact spots.

Application of the samples was done using nitrogen aspirator at a constant $150 \mathrm{nl} / \mathrm{s}$ application rate and spotted with first application position $X=10 \mathrm{~mm}$ and position $Y=10 \mathrm{~mm}$ in the form of distinct bands of length $5 \mathrm{~mm}$ with each spot being $11.4 \mathrm{~mm}$ apart. Linear ascending twin trough glass chamber (Camag Muttenz, Switzerland) of the dimensions $10 \times$ $10 \mathrm{~cm}$ saturated with mobile phase was used for linear ascending development of the plates. The optimized chamber saturation time for mobile phase was $20 \mathrm{~min}$ at room temperature and the length of chromatogram run was $85 \mathrm{~mm}$.

The plate was dried by air dryer. The plates were sprayed with anisaldehyde-sulphuric acid reagent comprising of $0.5 \mathrm{ml}$ anisaldehyde $+5 \mathrm{ml}$ sulphuric acid $+85 \mathrm{ml}$ methanol $+10 \mathrm{ml}$ glacial acetic acid with subsequent drying at $110^{\circ}$ in a hot air oven (10-15 min) till the appearance of dark pink spots. Slit dimensions of length $4.00 \mathrm{~mm}$ and thickness $0.45 \mathrm{~mm}$ with a $20 \mathrm{~mm} / \mathrm{s}$ scanning speed were employed and scanning of the dried chromatogram was done by Camag TLC scanner III in the reflectance mode $555 \mathrm{~nm}^{[15]}$. The radiation source employed was halogen tungsten lamp and Win CATS planar chromatography version 1.1.3.0 was used for its operation. The concentrations of chromatographed compounds were determined from the potency of dispersed reflected light. The peak areas with linear regression were determined ${ }^{[16-19]}$. 


\section{Calibration curve of forskolin:}

Methanol was used for preparing forskolin stock solution $(1000 \mu \mathrm{g} / \mathrm{ml})$, which was prior to spotting filtered through a membrane filter of thickness $0.25 \mu \mathrm{m}$. Spotting was performed in triplicate with different volumes ranging from 0.1 to $1.0 \mu \mathrm{l}$ on the HPTLC plate corresponding to concentrations of 100-1000 ng of forskolin and by linear least square regression analysis the peak area vs. drug concentration data was treated.

\section{Method validation:}

Linearity, precision, limit of detection (LOD) and limit of quantification (LOQ), robustness and accuracy were the parameters validated as per ICH guidelines ${ }^{[20]}$. A standard curve of forskolin was constructed with the concentration versus peak area plotted between 100$1000 \mathrm{ng} / \mathrm{spot}$.

Evaluation of precision was done with respect to intra- and inter-day precision. Two hundred and 600 $\mathrm{ng} / \mathrm{spot}$ were the two concentration levels of sample solution at which the inter- and intra-day variation were determined. The sample solutions (200 and 600 $\mathrm{ng} / \mathrm{spot}$ ) were analysed three times on the same day for intra-day variation study whereas for inter-day variation the sample solutions (200 and $600 \mathrm{ng} / \mathrm{spot}$ ) were analysed thrice each day for three consecutive days. The mean, standard deviation (SD) and \% relative standard deviation (\% RSD) values were calculated with the peak areas obtained.

Small changes in mobile phase composition and amount of mobile phase were the parameters primarily studied at concentration levels of 600 and $1000 \mathrm{ng} /$ spot to assess the robustness of the developed method. Changes in mobile phase composition involved small variations in the ratios of mobile phase. Ethyl acetate:hexane:formic acid of ratios (6.5:3.4:0.1 v/v and 7.5:2.4:0.1 v/v) were the mobile phases composition studied. Amount of mobile phase was another parameter for which the robustness study was carried out with two volumes of mobile phase 15 and $20 \mathrm{ml}$. The TLC plate was spotted with blank methanol six times for estimating the LOD and LOQ with subsequent determination of noise level. Signal-to-noise ratio of 3:1 is considered as LOD while 10:1 as LOQ. SD was determined for the analytical response and LOD and LOQ were thus experimentally verified. Samples previously analysed were added with an additional 50, 100 and $150 \%$ of the standard forskolin to determine the accuracy of the method. The testing was carried out in triplicate and the recovery of forskolin at different levels in the formulations was determined.

\section{Analysis of forskolin from the formulation:}

Quantification of drug from developed novel nanosuspension was performed using the proposed validated HPTLC method as previously described earlier. Single emulsion technique was employed for formulating forskolin nanosuspension. Polylactic glycolic acid (PLGA) and chitosan were the polymers primarily employed for the formulation process. Dichloromethane (DCM), was used as an organic solvent to solubilise the required amount of polymer (PLGA 50:50) and hence, constituted the organic phase while chitosan (mucoadhesive polymer) and polyvinyl alcohol, used as a stabilizer were dissolved in $1 \%$ acetic acid with $\mathrm{pH}$ adjusted ( $\mathrm{pH}$ 5.0) constituted the aqueous phase. The organic phase was emulsified in aqueous phase by means of probe sonicator for specified period of time. The resultant $\mathrm{O} / \mathrm{W}$ emulsion was kept on a magnetic stirrer for evaporating DCM at room temperature for 5-6 h till the solvent completely evaporated. The nanosuspension was centrifuged at $15000 \mathrm{rpm}$ for $30 \mathrm{~min}$ and was thrice washed to remove excess of stabilizer. The pellet obtained was redispersed in 2-3 ml water and was lyophilized to obtain freeze dried nanoparticles. Extraction of the nanoparticles obtained was done with $10 \mathrm{ml}$ methanol and for complete extraction of the drug, sonication for $30 \mathrm{~min}$ followed by centrifugation at $15000 \mathrm{rpm}$ for $15 \mathrm{~min}$ were performed. The supernatant obtained from the formulation was filtered and applied in triplicate on the TLC plate followed by development and scanning as described in the previous section. Other than analysis of drug content from the nanosuspension, the chances of excipients interference with the drug peak was also studied.

\section{Stability studies:}

The stability-indicating property of the HPTLC method developed was assessed by conducting forced degradation studies of forskolin according to ICH guidelines. Forskolin was subjected to acid and base hydrolysis, oxidation, photochemical degradation by UV and sunlight. Forskolin solution containing $50 \mathrm{mg} / 50 \mathrm{ml}$ of methanol was prepared as the stock solution, which was further used for stress degradation studies.

\section{Preparation of acid and base-induced degradation:}

To $5 \mathrm{ml}$ of $1 \mathrm{M} \mathrm{HCl}$ and $1 \mathrm{M} \mathrm{NaOH}, 5 \mathrm{ml}$ of stock solution 
was added separately and the mixtures were refluxed in different containers at $90^{\circ}$ for $6 \mathrm{~h}$ with the whole experiment performed in dark to avoid the possible side effects of light. $2 \mu 1$ of the resultant solution (800 ng/ spot) was applied on TLC plate and as per the method described in previous section the chromatograms were run.

\section{Preparation of hydrogen peroxide-induced degradation:}

To $5 \mathrm{ml}$ of hydrogen peroxide $\left(\mathrm{H}_{2} \mathrm{O}_{2} ; 30 \%\right.$, v/v), $5 \mathrm{ml}$ of stock solution was added in dark to eliminate the possible effects of light with the solution being refluxed for $6 \mathrm{~h}$ at $90^{\circ} .2 \mu \mathrm{l}$ of the resultant solution was applied smoothly on TLC plate as a single spot. The experimentation for chromatogram development and analysis was executed as per method described earlier.

\section{Sunlight and UV light-induced degradation:}

The stock solution of forskolin was exposed to direct sunlight and UV irradiation at $254 \mathrm{~nm}$ for $48 \mathrm{~h}$ to carry out photochemical-induced degradation, respectively. Diluted samples of the resultant solutions were properly prepared with the spots applied to the TLC plate and were analysed with the chromatogram run as per the method described previously.

\section{RESULTS AND DISCUSSION}

A HPTLC method for analysis of forskolin was developed with proper selection and optimization of the mobile phase. Compact and dense spots with considerably different $\mathrm{R}_{\mathrm{f}}$ values of the solvent system were particularly desired for forskolin. Various combinations of mobile phases were investigated and evaluated for HPTLC analysis of forskolin and finally plates were developed using a mobile phase of composition ethyl acetate:hexane:formicacid $(7: 2.9: 0.1 \mathrm{v} / \mathrm{v})$. Formic acid was added after thorough optimization since, before the addition of formic acid, tailing problem was observed in the chromatogram developed (fig. 2); however, after addition of $0.1 \mathrm{ml}$ of formic acid the problem was overcome and more compact spots, with normal symmetrical peak and $\mathrm{R}_{\mathrm{f}}$ value of 0.46 with good resolution for forskolin were obtained (fig. 2).

Calibration curves of forskolin $(n=3)$ studied over a range of 100-1000 ng/spot showed a good linear relationship with respect to peak area as shown in fig. 3 . The slopes of the standard curves showed no significant difference (ANOVA; $\mathrm{p}<0.05$ ). The graph was found

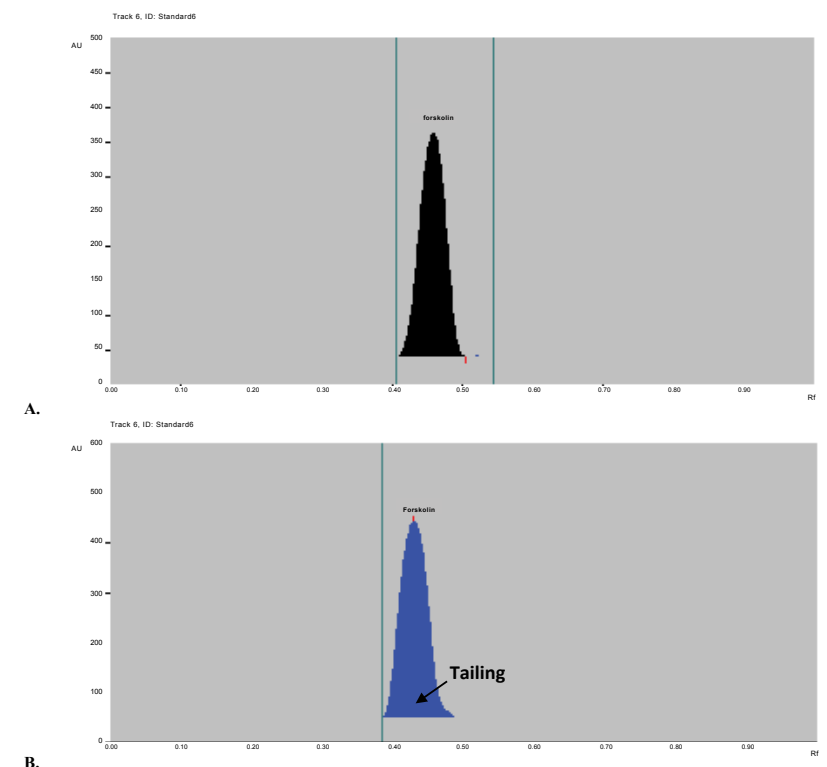

Fig. 2: HPTLC chromatogram

(A) Forskolin and (B) forskolin before addition of formic acid

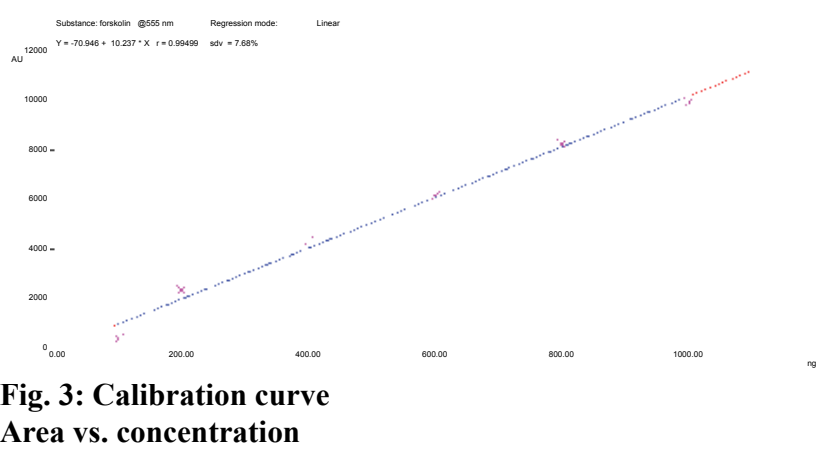

to be linear. A coefficient of correlation $\mathrm{r}^{2}=0.994$ was obtained for the calibration plots (100-1000 ng/spot), which indicated good linear relationship with slope and intercept values obtained as; slope $=10.237 \pm 1.27$ and intercept $=70.946 \pm 2.65 \quad(n=3)$ over the concentration range studied. The slopes of the standard curves in this range depicted no significant differences (ANOVA; $\mathrm{p}<0.05$ ). The linear regression data is as shown Table 1 .

Peak area measurement and sample application repeatability were expressed in terms of $\%$ RSD values. The $\%$ RSD for two concentration levels 400 and $600 \mathrm{ng} /$ spot was found to be 0.64 and 0.51 , respectively. Results for inter-day and intra-day variation of forskolin peak area for both concentrations are as shown in Table 2 . The $\%$ RSD values were found to be $<2 \%$ in all instances, which indicated that the developed method is of high precision.

Amount of the mobile phase and small changes in mobile phase were the parameters studied for determining robustness of the method. Percent RSD values were calculated for each parameter from the 
TABLE 1: LINEAR REGRESSION DATA FOR CALIBRATION PLOT FOR FORSKOLIN $(n=3)$

\begin{tabular}{lc}
\hline Parameters & Values \\
\hline Linearity range $(\mathrm{ng} / \mathrm{spot})$ & $100-1000 \mathrm{ng} / \mathrm{spot}$ \\
Regression equation & $\mathrm{Y}=-70.946+10.237 \times \mathrm{X}$ \\
Correlation coefficient & 0.994 \\
Slope $\pm \mathrm{SD}$ & $10.237 \pm 1.27$ \\
Intercept $\pm \mathrm{SD}$ & $70.946 \pm 2.65$ \\
$\mathrm{R}_{\mathrm{f}}$ value & $0.46 \pm 0.012$ \\
\hline
\end{tabular}

TABLE 2: INTRA- AND INTER-DAY PRECISION OF FORSKOLIN $(n=3)$

\begin{tabular}{lrrrc}
\hline $\begin{array}{l}\text { Amount } \\
\text { (ng/spot) }\end{array}$ & $\begin{array}{c}\text { Mean } \\
\text { (area) }\end{array}$ & SD (area) & $\begin{array}{c}\text { \% RSD } \\
\text { (area) }\end{array}$ & $\begin{array}{c}\text { SEM } \\
\text { (area) }\end{array}$ \\
\hline 200 & Intra-day & & & \\
\hline 600 & 2369.4 & 32.3529 & 1.36545 & 18.69 \\
& $\begin{array}{c}6161.5 \\
\text { Inter-day }\end{array}$ & 31.5013 & 0.51136 & 18.2 \\
\hline 200 & 2373.6 & 27.6892 & 1.16654 & 16 \\
600 & 6170.4 & 39.6752 & 0.64298 & 22.9 \\
\hline
\end{tabular}

TABLE 3: ROBUSTNESS OF FORSKOLIN $(n=3)$

\begin{tabular}{lcc}
\hline Forskolin & \multicolumn{2}{c}{ Mobile phase composition } \\
\hline Amount & $\begin{array}{c}\text { Ethyl } \\
\text { acetate:hexane:formic } \\
\text { acid }\end{array}$ & $\begin{array}{c}\text { Ethyl } \\
\text { acetate:hexane:formic } \\
\text { acid }\end{array}$ \\
(ng/spot) & $\begin{array}{c}(6.5: 3.4: 0.1, \mathrm{v} / \mathrm{v}) \% \\
\text { RSD (area) }\end{array}$ & $\begin{array}{c}(7.5: 2.4: 0.1, \mathrm{v} / \mathrm{v}) \% \\
\text { RSD (area) }\end{array}$ \\
600 & 0.89 & 0.55 \\
1000 & 0.36 & 0.23 \\
Forskolin & Amount of mobile phase $( \pm 5 \%)$ \\
\hline Amount & $15 \mathrm{ml}$ \% RSD (area) & $20 \mathrm{ml}, \%$ RSD (area) \\
(ng/spot) & 0.85 & 0.99 \\
600 & 0.54 & 0.53 \\
1000 & &
\end{tabular}

peak areas obtained and since it was less than $2 \%$, the developed method was found to be robust. Table 3 showed the respective results.

LOD is measured as 3:1 signal-to-noise ratio while LOQ as 10:1. The values for LOD and LOQ were found to be 10.83 and $36.12 \mathrm{ng} / \mathrm{spot}$, respectively. Fifty, 100 and $150 \%$ of the standard forskolin were added to previously analysed forskolin samples for carrying out a recovery study. The proposed TLC method afforded good recovery ranging from 99.04 to $100.24 \%$ as shown in Table 4, and indicated that the method was adequate. In Table 5, a summary of the validation parameters have been shown.

The chromatogram of the samples extracted from developed nanoparticulate formulation showed a single spot of $R_{f}$ value of 0.47 . The drug content i.e. amount of forskolin encapsulated in the formulation was found to be $94.1 \%$ with a \% RSD of 0.0357 for three replicates. The suitability of the method is indicated by low value of \% RSD and thus, can be used for routine analysis of forskolin in various dosage forms. No degradation of the drug occurred in formulation as the developed chromatogram showed a single peak of drug with little or no interference of the excipients during the analysis.

Additional peaks at different $R_{f}$ values were obtained for the chromatograms of the acid-degraded samples and base-degraded samples. Three peaks for acidinduced degraded sample were observed at $R_{f}$ values $0.12,0.37,0.39$ as shown in Table 6 and fig. 4 a while for base-induced degraded sample two peaks were observed at $R_{f}$ values 0.02 and 0.57 as shown in Table 6 and fig. 4b. The forskolin spot was well resolved from the degraded products from acid and base-induced degradation. The three additional peaks apart from forskolin peak were obtained as degraded product peaks from hydrogen peroxide $30 \% \mathrm{v} / \mathrm{v}$ treatment

TABLE 4: RECOVERY STUDIES $(n=3)$

\begin{tabular}{lcccc}
\hline $\begin{array}{l}\text { Excess drug } \\
\text { added to } \\
\text { analyte (\%) }\end{array}$ & $\begin{array}{c}\text { Theoretical } \\
\text { content (ng) }\end{array}$ & $\begin{array}{c}\text { Recovery } \\
(\%, \text { area) }\end{array}$ & $\begin{array}{c}\text { RSD } \\
\text { (\%, area) }\end{array}$ & $\begin{array}{c}\text { SEM } \\
\text { (area) }\end{array}$ \\
\hline 0 & 400 & 99.04 & 1.69 & 42.06 \\
50 & 600 & 99.83 & 0.16 & 5.25 \\
100 & 800 & 100.24 & 0.24 & 11.56 \\
150 & 1000 & 100.13 & 0.41 & 23.97 \\
\hline
\end{tabular}

\begin{tabular}{|c|c|c|}
\hline $\begin{array}{lcl}\text { TABLE 5: SUMMARY } \\
\text { PARAMETERS }\end{array}$ & OF & VALIDATION \\
\hline Parameter data & & Data \\
\hline Linearity range (ng/spot) & 100 & $1000 \mathrm{ng} / \mathrm{spot}$ \\
\hline Correlation coefficient & & 0.994 \\
\hline Limit of detection & 10.8 & 33 ng per spot \\
\hline Limit of quantification & 36. & 2 ng per spot \\
\hline Recovery studies (accuracy, $n=3$ ) & $99 .($ & 4 to $100.24 \%$ \\
\hline Robustness & & Robust \\
\hline \multicolumn{3}{|l|}{ Precision (\% RSD) } \\
\hline Inter-day $(n=3)$ & & 0.64 \\
\hline Intra-day $(n=3)$ & & 0.51 \\
\hline
\end{tabular}

TABLE 6: DEGRADATION PRODUCTS OF FORSKOLIN WITH $R_{f}$ VALUES

\begin{tabular}{lccc}
\hline $\begin{array}{l}\text { Stress } \\
\text { condition }\end{array}$ & $\begin{array}{c}\text { No. of degradation } \\
\text { products } \mathbf{R}_{\mathrm{f}} \text { value }\end{array}$ & $\begin{array}{c}\text { Forskolin } \\
\mathbf{R}_{\mathrm{f}} \text { value }\end{array}$ & $\begin{array}{c}\% \\
\text { Recovery }\end{array}$ \\
\hline $\begin{array}{l}\text { Acid }(1 \mathrm{M} \mathrm{HCl}) \\
\text { Base }\end{array}$ & $3(0.12,0.27,0.39)$ & 0.51 & 2.85 \\
$(1 \mathrm{M} \mathrm{NaOH})$ & $2(0.02,0.57)$ & 0.48 & 5.65 \\
$\begin{array}{l}\text { Peroxide } \\
\left(30 \% \mathrm{H}_{2} \mathrm{O}_{2}\right)\end{array}$ & $3(0.23,0.39,0.75)$ & 0.5 & 69.4 \\
$\begin{array}{l}\text { Sunlight } \\
\text { UV light }\end{array}$ & $\begin{array}{c}(0.54,0.64,0.75) \\
(0.12,0.32,0.40,\end{array}$ & 0.47 & 77.2 \\
\hline
\end{tabular}



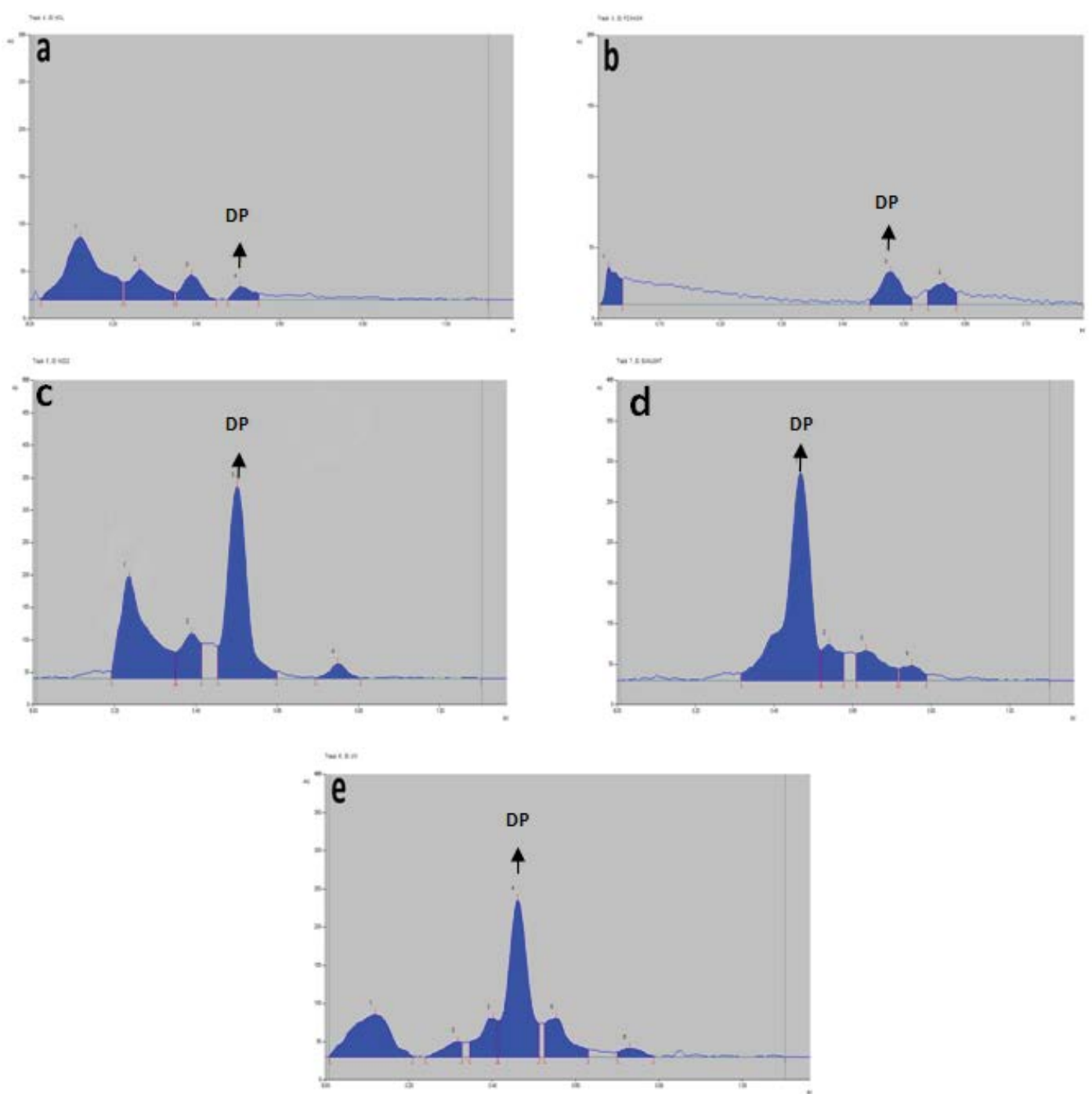

Fig. 4: Forced degradation of forskolin

(a) Acid degradation (b) base degradation (c) $\mathrm{H}_{2} \mathrm{O}_{2}$ degradation (d) sunlight degradation (e) UV light degradation. "DP indicates drug peak

at different $R_{\mathrm{f}}$ values, $0.23,0.39,0.75$ as depicted in Table 6 and fig. 4c. Forskolin spot was well resolved from among the degraded products.

Chromatograms for sunlight and UV-induced degraded products were obtained with variable peaks at different $\mathrm{R}_{\mathrm{f}}$ values. Three peaks for sunlight-induced degradation products at $\mathrm{R}_{\mathrm{f}}$ values $0.54,0.64,0.75$ as depicted in Table 6 and fig. $4 \mathrm{~d}$ whereas five peaks for UV-induced degradation products at $R_{f}$ values $0.12,0.32,0.40$, $0.56,0.73$ were observed as shown in Table 6 and fig. $4 \mathrm{e}$ with the drug spots for respective parameters well resolved from the other peaks (degraded products).

In conclusion, after thorough optimization and validation, a validated HPTLC method for the analysis of forskolin has been developed. The developed method was found to be simple, precise, accurate, less time consuming and cost-effective, reproducible and selective as evidenced by the statistical analysis of data and could be used for routine analysis of forskolin. As compared to other densitometric methods, the developed HPTLC method has been found to be more accurate since it has been properly optimized, developed and validated. Purity of the drug can be assessed by this method as the drug is available from a variety of sources and detection of associated impurities can also be done with developed approach. Statistical analysis has proved that the method is repeatable, accurate and precise and therefore, can be employed for analysis of forskolin in various formulations. No degradation of drug occurred in formulation and least interaction of excipients occurred as single chromatogram of forskolin was observed with $R_{f}$ value of 0.47. Quantitative estimation of the said drug 
i.e., forskolin can also be done in plasma and other biological fluids. Prediction of shelf life of forskolin containing pharmaceuticals by accelerated stability testing can also be done by employing the developed method. Forskolin can be well effectively used in the formulation of nano-dosage forms and can be used for glaucoma treatment in future.

\section{Acknowledgements:}

Nazia Khan thanks the Indian Council of Medical Research (ICMR), New Delhi, India for providing a Senior Research Fellowship (File No. 45/25/2013NAN/BMS). The authors are also grateful to Sami Labs, Bangalore, India for the gift sample of forskolin. Authors wish to thank Dr. Mohd Yawar Ali Khan for his help and support.

\section{Conflicts of interest:}

There are no conflicts of interest.

\section{REFERENCES}

1. Sahoo N, Manchikanti P, Dey S. Herbal drugs: Standards and regulation. Fitoterapia 2010;81(6):462-71.

2. Yamamura K, Nakao M, Yano K, Miyamoto Ki, Yotsuyanagi T. Stability of forskolin in lipid emulsions and oil/water partition coefficients. Chem Pharm Bull 1991;39:1032-4.

3. Caprioli J, Sears M, Bausher L, Gregory D, Mead A. Forskolin lowers intraocular pressure by reducing aqueous inflow. Invest Ophthalmol Vis Sci 1984;25:268-77.

4. Burstein NL, Sears ML, Mead A. Aqueous flow in human eyes is reduced by forskolin, a potent adenylate cyclase activator. Exp Eye Res 1984;39:745-9.

5. Gupta S, Samanta MK, Raichur AM. Dual-drug delivery system based on in situ gel-forming nanosuspension of forskolin to enhance antiglaucoma efficacy. AAPS PharmSciTech 2010;11:322-35.

6. Smith BR, Gaster RN, Leopold IH, Zeleznick LD. Forskolin, a potent adenylate cyclase activator, lowers rabbit intraocular pressure. Arch Ophthalmol 1984;102:146-8.

7. Kumarasamy NA, Lam FS, Wang AL, Theoharides TC. Glaucoma: Current and developing concepts for inflammation, pathogenesis and treatment. Eur J Inflamm 2006;4:129-37.
8. Variyar PS, Chatterjee S, Sharma A. Fundamentals and Theory of HPTLC-Based Separation. In: MM. Srivastava, editor. High-Performance Thin-Layer Chromatography (HPTLC). Heidelberg, Germany: Springer Verlag GmbH; 2011. p. 27-39.

9. Ahmad S, Rizwan M, Parveen R, Mujeeb M, Aquil M. A validated stability-indicating TLC method for determination of forskolin in crude drug and pharmaceutical dosage form. Chromatographia 2008;67:441-7.

10. Dwivedi BK, Kumar S, Kumar A. Analysis of forskolin in Homoeopathic tinctures by validated HPLC method. Int $\mathrm{J}$ Med Arom Plants 2012;2:195-9.

11. Blessy MR, Patel RD, Prajapati PN, Agrawal YK. Development of forced degradation and stability indicating studies of drugs-A review. J Pharm Anal 2014;4:159-65.

12. Reviewer Guidance: Validation of chromatographic methods. Center for Drug Evaluation and Research (CDER), Food and Drug Administration (FDA). Available from: https://www.fda. gov/downloads/drugs/guidances/ucm134409.pdf.

13. Analytical Procedures and Methods Validation for Drugs and Biologics. Guidance for Industry. Available from: https:// www.fda.gov/downloads/drugs/guidances/ucm386366.pdf.

14. Guidance for Industry. INDs for Phase 2 and 3 Studies. Available from: https://www.fda.gov/downloads/Drugs/ Guidances/ucm070567.pdf.

15. Guidance for Industry: Q2B Validation of Analytical Procedures: Methodology. Available form: https://www.fda. gov/downloads/drugs/guidances/ucm073384.pdf.

16. ICH Harmonised Tripartite Guideline. Guidance on Analytical Method Validation. Available form: https://www.ich.org/ fileadmin/Public Web Site/ICH Products/Guidelines/ Quality/Q2_R1/Step4/Q2_R1_Guideline.pdf.

17. Agrawal H, Kaul N, Paradkar AR, Mahadik KR. HPTLC method for guggul sterone: I. Quantitative determination of E-and Z-guggul sterone in herbal extract and pharmaceutical dosage form. J Pharm Biomed Anal 2004;36:33-41.

18. Ameeduzzafar, Ali J, Ali A. Stability-indicating HPTLC method of carteolol in bulk drug and in pharmaceutical dosage forms. J Plan Chromatogr 2013;26:86-92.

19. Mahadik KR, Paradkar AR, Agrawal H, Kaul N. Stabilityindicating HPTLC determination of tizanidine hydrochloride in bulk drug and pharmaceutical formulations. J Pharm Biomed Anal 2003;33:545-52.

20. Tayade NG, Nagarsenker MS. Validated HPTLC method of analysis for artemether and its formulations. J Pharm Biomed Anal 2007;43:839-44. 